\title{
ON THE KOBAYASHI HYPERBOLICITY OF CERTAIN TUBE DOMAINS
}

\author{
ALAN HUCKLEBERRY AND ALEXANDER ISAEV
}

(Communicated by Franc Forstneric)

\begin{abstract}
In an article from 2008 the second author introduced three families of tube domains in $\mathbb{C}^{2}$ with holomorphic automorphism group isomorphic to $\mathbb{R} \ltimes \mathbb{R}^{2}$ and envelope of holomorphy equal to $\mathbb{C}^{2}$. In the present paper we show that every domain in each of these families is Kobayashi-hyperbolic.
\end{abstract}

\section{INTRODUCTION}

A connected complex manifold $X$ is called Kobayashi-hyperbolic if the Kobayashi pseudodistance on $X$ is in fact a distance (see $[\mathrm{K}]$ for details). If $X$ is equipped with a Riemannian metric, the hyperbolicity property can be stated as follows: for any point $x \in X$ there exist a neighborhood $U$ of $x$ and a constant $M>0$ such that for all holomorphic maps $f: \Delta \rightarrow X$ with $f(0) \in U$ one has $\|d f(0)\|<M$, where $\Delta$ is the unit disk in $\mathbb{C}$ (see, e.g., $[\mathrm{L}]$ ). Verification of hyperbolicity for a particular manifold may be a difficult task. In this paper we show that certain explicitly given tube domains in $\mathbb{C}^{2}$ are hyperbolic.

Recall that a tube domain in $\mathbb{C}^{n}$ is a domain of the form $T_{D}:=D+i \mathbb{R}^{n}$, where $D$ is a domain in $\mathbb{R}^{n}$ called the base of $T_{D}$. By Bochner's theorem, the envelope of holomorphy of $T_{D}$ coincides with $T_{\widehat{D}}$, where $\widehat{D}$ is the convex hull of $D$ in $\mathbb{R}^{n}$ (see, e.g., Section 21 in $[\mathrm{V}]$ ). For tube domains in $\mathbb{C}^{2}$ with $T_{\widehat{D}} \neq \mathbb{C}^{2}$, a hyperbolicity criterion was given in $[\mathrm{L}$. However, there is no reasonable sufficient condition for $T_{D}$ to be hyperbolic in the case $T_{\widehat{D}}=\mathbb{C}^{2}$. All tube domains considered in this paper fall into this last case. In particular, they do not admit any non-constant bounded holomorphic functions.

We will now introduce three families of domains in $\mathbb{R}^{2}$ as follows:

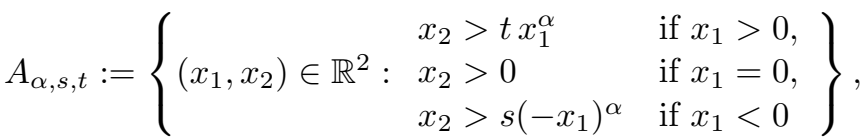

$$
\begin{aligned}
& \alpha>0, \alpha \neq 1, s<0, t>0,
\end{aligned}
$$

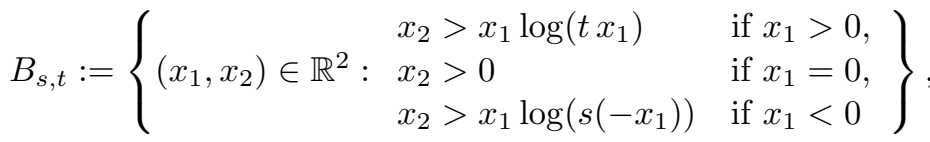

$$
\begin{aligned}
& s>0, t>0,
\end{aligned}
$$

Received by the editors November 14, 2011.

2010 Mathematics Subject Classification. Primary 32Q45, 31A15.

(C) 2013 American Mathematical Society 


$$
\begin{gathered}
C_{\alpha, s, t}:=\left\{\left(x_{1}, x_{2}\right) \in \mathbb{R}^{2}: s e^{\alpha \varphi}<r<t e^{\alpha \varphi}\right\}, \\
\alpha>0, t>0, e^{-2 \pi \alpha} t<s<t,
\end{gathered}
$$

where $(r, \varphi)$ are the polar coordinates in $\mathbb{R}^{2}$ with $\varphi$ varying from $-\infty$ to $\infty$.

If $X$ is hyperbolic, then the group $\operatorname{Aut}(X)$ of its holomorphic automorphisms can be given the structure of a (real) Lie group in the compact-open topology (for a discussion of this property in a more general setting, see Section 1.1 in [I1]). In I2 all hyperbolic manifolds $X$ with $\operatorname{dim}_{\mathbb{C}} X=2, \operatorname{dim}_{\mathbb{R}} \operatorname{Aut}(X)=3$ were classified up to biholomorphic equivalence, and the families of tube domains $\left\{T_{A_{\alpha, s, t}}\right\},\left\{T_{B_{s, t}}\right\},\left\{T_{C_{\alpha, s, t}}\right\}$ form part of the classification (they correspond to the domains listed in II2 under the headings $(\mathbf{1})(\mathbf{c}),(\mathbf{2})(\mathbf{b}),(5)$, respectively). The holomorphic automorphism group of each of these tube domains is isomorphic to $\mathbb{R} \ltimes_{\rho} \mathbb{R}^{2}$ for some homomorphism $\rho: \mathbb{R} \rightarrow \mathrm{GL}(2, \mathbb{R})$. For all manifolds that appear in the classification other than the tube domains in the families $\left\{T_{A_{\alpha, s, t}}\right\},\left\{T_{B_{s, t}}\right\}$, $\left\{T_{C_{\alpha, s, t}}\right\}$, verification of hyperbolicity is straightforward and therefore was omitted in [12]. In contrast, ascertaining the hyperbolicity of domains in $\left\{T_{A_{\alpha, s, t}}\right\},\left\{T_{B_{s, t}}\right\}$, $\left\{T_{C_{\alpha, s, t}}\right\}$ is non-trivial. However, no statement confirming hyperbolicity for such domains was given in [12] either. In the present paper we address this issue by proving the following theorem.

Theorem 1.1. Every domain in each of the families $\left\{T_{A_{\alpha, s, t}}\right\},\left\{T_{B_{s, t}}\right\},\left\{T_{C_{\alpha, s, t}}\right\}$ is hyperbolic.

In addition to supplementing the arguments of [12], the proof of Theorem 1.1 given in the next section is also of independent interest since it in fact applies to a much larger class of tube domains satisfying the condition $T_{\widehat{D}}=\mathbb{C}^{2}$ (see Remark 2.3).

\section{Proof of Theorem 1.1}

As pointed out in $\mathrm{L}$, for a tube domain $T_{D} \subset \mathbb{C}^{n}$ the hyperbolicity property is equivalent to the following condition: for any point $x \in D$ there exist a neighborhood $U$ of $x$ in $D$ and a constant $M>0$ such that for all harmonic maps $f: \Delta \rightarrow D$ with $f(0) \in U$ one has $\|d f(0)\|<M$. Hence $T_{D}$ is not hyperbolic if and only if there exist a point $a \in D$ and a sequence $\left\{f_{k}\right\}$ of harmonic maps from $\Delta$ into $D$ such that $f_{k}(0) \rightarrow a$ and $\left\|d f_{k}(0)\right\| \rightarrow \infty$ as $k \rightarrow \infty$.

Now let $D$ be a domain in one of the families $\left\{A_{\alpha, s, t}\right\},\left\{B_{s, t}\right\},\left\{C_{\alpha, s, t}\right\}$. Assuming that $T_{D}$ is not hyperbolic, we obtain a point $a=\left(a_{1}, a_{2}\right) \in D$ and a sequence $\left\{f_{k}\right\}$ as above, with $f_{k}=\left(u_{k}, v_{k}\right)$, where $u_{k}, v_{k}$ are real-valued harmonic functions on $\Delta$. In our proof of the theorem we utilize level sets of $u_{k}$. Some fundamental properties of such sets are given in the following proposition.

Proposition 2.1. For every $c \in \mathbb{R}$ one has:

(i) there exists $K \in \mathbb{N}$ such that $c \in u_{k}(\Delta)$ for all $k \geq K$,

(ii) $\operatorname{dist}\left(0, L_{k}(c)\right) \rightarrow 0$ as $k \rightarrow \infty$, where $L_{k}(c):=\left\{z \in \Delta: u_{k}(z)=c\right\}$.

Proof. We first prove statement (i). Assuming it is false, we obtain a subsequence $\left\{u_{k_{\ell}}\right\}$ of the sequence $\left\{u_{k}\right\}$ such that for some $c \neq a_{1}$ one has either $u_{k_{\ell}}<c$ in $\Delta$ (if $a_{1}<c$ ) or $u_{k_{\ell}}>c$ in $\Delta$ (if $a_{1}>c$ ) for all $k_{\ell}$. Then $f_{k_{\ell}}(\Delta)$ is contained in either $D_{-}(c):=D \cap\left\{x_{1}<c\right\}$ or $D_{+}(c):=D \cap\left\{x_{1}>c\right\}$ for all $k_{\ell}$, respectively.

Suppose first that $D$ belongs to the family $\left\{C_{\alpha, s, t}\right\}$. In this case the open sets $D_{-}(c)$ and $D_{+}(c)$ are disconnected and each of their countably many connected 
components is bounded. Clearly, a tube domain having a bounded base is hyperbolic. On the other hand, let $D^{\prime}(c)$ be the connected component containing the point $a$. Then $f_{k_{\ell}}(\Delta) \subset D^{\prime}(c)$ for large $k_{\ell}$, which contradicts the hyperbolicity of $T_{D^{\prime}(c)}$.

Suppose next that $D$ belongs to one of the families $\left\{A_{\alpha, s, t}\right\},\left\{B_{s, t}\right\}$. In this case $D_{-}(c)$ and $D_{+}(c)$ are connected. We will now show that the tube domains $T_{D_{-}(c)}$ and $T_{D_{+}(c)}$ are hyperbolic, thus contradicting the fact that $f_{k_{\ell}}(\Delta)$ is contained in either $D_{-}(c)$ or $D_{+}(c)$ for all $k_{\ell}$. We use the following well-known result.

Lemma $2.2(\mathrm{E}])$. Let $X, Y$ be complex manifolds and $F: X \rightarrow Y$ a holomorphic map. Suppose that $Y$ is hyperbolic and has an open cover $\left\{U_{\alpha}\right\}$ such that $F^{-1}\left(U_{\alpha}\right)$ is hyperbolic for every $\alpha$. Then $X$ is hyperbolic.

One now easily observes that $T_{D_{-}(c)}$ (resp., $\left.T_{D_{+}(c)}\right)$ is hyperbolic by choosing in Lemma 2.2 the manifold $Y$ to be $\left\{\left(z_{1}, 0\right) \in \mathbb{C}^{2}: \operatorname{Re} z_{1}<c\right\}$ (resp., $\left\{\left(z_{1}, 0\right) \in\right.$ $\left.\mathbb{C}^{2}: \operatorname{Re} z_{1}>c\right\}$ ), the open cover to be $\left\{\left(z_{1}, 0\right) \in \mathbb{C}^{2}: c-m<\operatorname{Re} z_{1}<c\right\}$ (resp., $\left.\left\{\left(z_{1}, 0\right) \in \mathbb{C}^{2}: c<\operatorname{Re} z_{1}<c+m\right\}\right), m \in \mathbb{N}$, and the map $F$ to be the projection to the $z_{1}$-coordinate complex line. This completes the proof of statement (i).

We will now prove statement (ii). Assuming it is false, we obtain a subsequence $\left\{f_{k_{\ell}}\right\}$ of the sequence $\left\{f_{k}\right\}$ and a disk $\Delta_{r}$ of radius $0<r<1$ centered at the origin such that for some $c \neq a_{1}$, the set $f_{k_{\ell}}\left(\Delta_{r}\right)$ is contained in either $D_{-}(c)$ (if $a_{1}<c$ ) or $D_{+}(c)$ (if $a_{1}>c$ ) for all $k_{\ell}$. Considering the sequence $\left\{\widetilde{f}_{k_{\ell}}\right\}$ of maps from $\Delta$ to $D$ defined by $\widetilde{f}_{k_{\ell}}(z):=f_{k_{\ell}}(r z)$ for $|z|<1$, we obtain a contradiction as in the proof of statement (i) above. The proof of Proposition 2.1 is complete.

In the remaining part of the proof of the theorem we will separately consider two cases.

Case 1. Suppose that $D$ belongs to one of the families $\left\{A_{\alpha, s, t}\right\},\left\{B_{s, t}\right\}$. Fix $R>0$, $p>0, q>0,0<\varepsilon<1$ such that $p R>a_{1}, q R>-a_{1}$ and consider the open set

$$
\left\{z \in \Delta: a_{1}-p R<u_{k}(z)<a_{1}+q R,|z|<1-\varepsilon\right\} .
$$

For all sufficiently large $k$ the origin lies in this set, and we denote by $\Omega_{k}$ its connected component containing the origin. By the maximum principle for harmonic functions, $\Omega_{k}$ is a Jordan simply connected domain, and we have

$$
\partial \Omega_{k}=\Gamma_{k} \sqcup \Gamma_{k}^{\prime} \sqcup \gamma_{k},
$$

where $\Gamma_{k} \subset L_{k}\left(a_{1}-p R\right), \Gamma_{k}^{\prime} \subset L_{k}\left(a_{1}+q R\right)$, and $\gamma_{k}:=\partial \Omega_{k} \cap\{|z|=1-\varepsilon\}$.

For a subset $E \subset \partial \Omega_{k}$, let $\omega_{k}(E)$ be the harmonic measure of $E$ at the origin associated to $\Omega_{k}$. By a well-known estimate (see Theorem IV.6.2 on p. 149 in [GM]) and Proposition 2.1, for any sufficiently large $k$ one has

$$
\omega_{k}\left(\gamma_{k}\right) \leq \frac{8}{\pi} \sqrt{\frac{\operatorname{dist}\left(0, \partial \Omega_{k}\right)}{1-\varepsilon}}
$$

which implies

$$
\omega_{k}\left(\gamma_{k}\right) \rightarrow 0 \quad \text { as } k \rightarrow \infty
$$


Next, let $\mu_{k}:=\omega_{k}\left(\Gamma_{k}\right)$ and $\mu_{k}^{\prime}:=\omega_{k}\left(\Gamma_{k}^{\prime}\right)$. We have

$$
\begin{aligned}
u_{k}(0) & =\int_{\Gamma_{k}} u_{k} d \omega_{k}+\int_{\Gamma_{k}^{\prime}} u_{k} d \omega_{k}+\int_{\gamma_{k}} u_{k} d \omega_{k} \\
& =\left(a_{1}-p R\right) \mu_{k}+\left(a_{1}+q R\right) \mu_{k}^{\prime}+\int_{\gamma_{k}} u_{k} d \omega_{k} .
\end{aligned}
$$

Since on $\gamma_{k}$ the function $u_{k}$ is bounded from above and below by constants independent of $k$, from (2.1) we obtain that the last summand in (2.2) tends to zero as $k \rightarrow \infty$. Thus, (2.1) and (2.2) yield

$$
\begin{aligned}
& \mu_{k}+\mu_{k}^{\prime} \rightarrow 1, \\
& \left(a_{1}-p R\right) \mu_{k}+\left(a_{1}+q R\right) \mu_{k}^{\prime} \rightarrow a_{1}, \quad \text { as } k \rightarrow \infty,
\end{aligned}
$$

which implies

$$
\mu_{k} \rightarrow \frac{q}{p+q}, \quad \mu_{k}^{\prime} \rightarrow \frac{p}{p+q} \quad \text { as } k \rightarrow \infty .
$$

We will now consider two situations.

Case 1a. Assume that $D=A_{\alpha, s, t}$ for some $\alpha>0, \alpha \neq 1, s<0, t>0$. Then

$$
\begin{aligned}
& v_{k}(z)>s\left(p R-a_{1}\right)^{\alpha} \text { for } z \in \Gamma_{k}, \gamma_{k}, \\
& v_{k}(z)>t\left(a_{1}+q R\right)^{\alpha} \text { for } z \in \Gamma_{k}^{\prime} .
\end{aligned}
$$

Therefore, we have

$$
\begin{aligned}
v_{k}(0) & =\int_{\Gamma_{k}} v_{k} d \omega_{k}+\int_{\Gamma_{k}^{\prime}} v_{k} d \omega_{k}+\int_{\gamma_{k}} v_{k} d \omega_{k} \\
& \geq s\left(p R-a_{1}\right)^{\alpha} \mu_{k}+t\left(a_{1}+q R\right)^{\alpha} \mu_{k}^{\prime}+s\left(p R-a_{1}\right)^{\alpha} \omega_{k}\left(\gamma_{k}\right) .
\end{aligned}
$$

Letting in the above inequality $k \rightarrow \infty$ and using (2.1), (2.3), we then obtain

$$
\begin{aligned}
a_{2} \geq s\left(p R-a_{1}\right)^{\alpha} \frac{q}{p+q}+ & t\left(a_{1}+q R\right)^{\alpha} \frac{p}{p+q} \\
= & \frac{R^{\alpha}}{p+q}\left[s q\left(p-\frac{a_{1}}{R}\right)^{\alpha}+t p\left(q+\frac{a_{1}}{R}\right)^{\alpha}\right] .
\end{aligned}
$$

Choosing $p, q$ such that $(q / p)^{\alpha-1}>|s| / t$ and letting $R \rightarrow \infty$, we now observe that the right-hand side of (2.5) can be made arbitrarily large. This contradiction concludes the proof of the theorem in the case when $D$ belongs to the family $\left\{A_{\alpha, s, t}\right\}$.

Case $1 \mathrm{~b}$. Assume that $D=B_{s, t}$ for some $s>0, t>0$. Then

$$
\begin{aligned}
& v_{k}(z)>\left(a_{1}-p R\right) \log \left(s\left(p R-a_{1}\right)\right) \text { for } z \in \Gamma_{k}, \\
& v_{k}(z)>\left(a_{1}+q R\right) \log \left(t\left(a_{1}+q R\right)\right) \text { for } z \in \Gamma_{k}^{\prime}, \\
& v_{k}(z)>C \text { for } z \in \gamma_{k}, \text { where } C \text { is a constant independent of } k .
\end{aligned}
$$

Therefore, analogously to (2.4) we have

$$
v_{k}(0) \geq\left(a_{1}-p R\right) \log \left(s\left(p R-a_{1}\right)\right) \mu_{k}+\left(a_{1}+q R\right) \log \left(t\left(a_{1}+q R\right)\right) \mu_{k}^{\prime}+C \omega_{k}\left(\gamma_{k}\right) .
$$


Letting in the above inequality $k \rightarrow \infty$ and using (2.1), (2.3), we then obtain

$$
\begin{aligned}
a_{2} \geq\left(a_{1}-p R\right) \log \left(s\left(p R-a_{1}\right)\right) \frac{q}{p+q}+ & \left(a_{1}+q R\right) \log \left(t\left(a_{1}+q R\right)\right) \frac{p}{p+q} \\
=\frac{R}{p+q}\left[q\left(\frac{a_{1}}{R}-p\right) \log \left(s\left(p R-a_{1}\right)\right)\right. & \\
& \left.+p\left(\frac{a_{1}}{R}+q\right) \log \left(t\left(a_{1}+q R\right)\right)\right] .
\end{aligned}
$$

Choosing $p, q$ such that $t q>s p$ and letting $R \rightarrow \infty$, we now observe that the right-hand side of (2.6) can be made arbitrarily large. This contradiction concludes the proof of the theorem in the case when $D$ belongs to the family $\left\{B_{s, t}\right\}$.

Case 2. Now suppose that $D$ belongs to the family $\left\{C_{\alpha, s, t}\right\}$. Fix $c>a_{1}, 0<\varepsilon<1$ and consider the open set

$$
\left\{z \in \Delta: u_{k}(z)<c,|z|<1-\varepsilon\right\} .
$$

For all sufficiently large $k$ the origin lies in this set, and we denote by $\Omega_{k}$ its connected component containing the origin. As in Case $1, \Omega_{k}$ is a Jordan simply connected domain, and we have

$$
\partial \Omega_{k}=\Gamma_{k} \sqcup \gamma_{k},
$$

where $\Gamma_{k} \subset L_{k}(c)$ and $\gamma_{k}:=\partial \Omega_{k} \cap\{|z|=1-\varepsilon\}$.

Recall from the proof of Proposition 2.1 that the open set $D_{-}(c)=D \cap\left\{x_{1}<c\right\}$ has countably many connected components and each component is bounded. Let $D^{\prime}(c)$ be the connected component of $D_{-}(c)$ containing $a$. Clearly, $f_{k}\left(\Omega_{k}\right) \subset D^{\prime}(c)$ for all sufficiently large $k$. This implies that on $\gamma_{k}$ the function $u_{k}$ is bounded from below by a constant independent of $k$ if $k$ is sufficiently large.

For a subset $E \subset \partial \Omega_{k}$, we let $\omega_{k}(E)$ be the harmonic measure of $E$ at the origin associated to $\Omega_{k}$ and $\mu_{k}:=\omega_{k}\left(\Gamma_{k}\right)$. Arguing as in Case 1, we then see that (2.1) holds; that is, $\mu_{k} \rightarrow 1$ as $k \rightarrow \infty$.

Next, we have

$$
u_{k}(0)=\int_{\Gamma_{k}} u_{k} d \omega_{k}+\int_{\gamma_{k}} u_{k} d \omega_{k}=c \mu_{k}+\int_{\gamma_{k}} u_{k} d \omega_{k}
$$

Since on $\gamma_{k}$ the function $u_{k}$ is bounded from above and below by constants independent of $k$, from (2.1) we obtain that the last summand in (2.7) tends to zero as $k \rightarrow \infty$. Thus, (2.7) implies $a_{1}=c$, which contradicts our choice of $c$. This completes the proof of the theorem.

Remark 2.3. The proof of Theorem 1.1 in fact applies to more general domains. Indeed, let $D$ be a domain of the form $\left\{\left(x_{1}, x_{2}\right): x_{2}>h\left(x_{1}\right)\right\}$, where $h \in C(\mathbb{R})$ and satisfies the following property: for every $b \in \mathbb{R}$ there exist $p>0, q>0$ such that

$$
q h(b-p R)+p h(b+q R) \rightarrow \infty \text { as } R \rightarrow \infty .
$$

Then the argument given for Case 1 yields that $T_{D}$ is hyperbolic. Next, let $D$ be a domain bounded by two general spirals, where a spiral is a curve defined by the equation $r=g(\varphi)$, with $g$ being an increasing function of $\varphi$ such that $\lim _{\varphi \rightarrow-\infty} g(\varphi)=0$ and $\lim _{\varphi \rightarrow+\infty} g(\varphi)=\infty$. Then the argument given for Case 2 shows that $T_{D}$ is hyperbolic. 
Remark 2.4. Before attempting to prove Theorem 1.1 in full generality, we set out to show that the domain $T_{A_{3,-1,1}}$ is Brody hyperbolic (recall that $A_{3,-1,1}=$ $\left.\left\{\left(x_{1}, x_{2}\right) \in \mathbb{R}^{2}: x_{2}>x_{1}^{3}\right\}\right)$. Brody hyperbolicity for a tube domain is equivalent to the non-existence of a non-constant harmonic map $f=(u, v)$ from the plane into the base of the domain (cf. [L]). Regarding this question, F. Nazarov suggested that we consider the connected component $\Omega(R, \rho)$ containing the origin of the open set

$$
\{z \in \mathbb{C}:-R<u(z)<2 R,|z|<\rho\}
$$

(assuming without loss of generality that $u(0)=0$ ), where $R>0$ and $\rho>0$ are large. Then the harmonic measure at the origin of the portion of $\partial \Omega(R, \rho)$ where $|z|=\rho$ tends to 0 as $\rho \rightarrow \infty$, and letting $R \rightarrow \infty$, one obtains that $v(0)$ is estimated from below by an arbitrarily large number. With F. Nazarov's kind permission, we used a similar approach in Case 1 of our proof of Theorem 1.1. We also point out that the idea to consider harmonic measures associated to domains bounded by level sets of $u$ was independently suggested to us by E. Poletsky and L. Kovalev.

\section{ACKNOWLEDGEMENTS}

The authors are indebted to F. Nazarov, E. Poletsky and L. Kovalev for communicating to them an idea that turned out to be instrumental for the proof of Theorem 1.1 (see Remark 2.4 for details). This work was initiated during the first author's visit to the Australian National University in September 2011. We gratefully acknowledge support of the Australian Research Council.

\section{REFERENCES}

[E] Eastwood, A., À propos des variétés hyperboliques complètes, C. R. Acad. Sci. Paris Sér. $A-B 280$ (1975), A1071-A1074. MR0414941 (54:3033)

[GM] Garnett, J. B. and Marshall, D. E., Harmonic Measure, New Mathematical Monographs, 2, Cambridge University Press, Cambridge, 2005. MR2150803 (2006g:31002)

[I1] Isaev, A. V., Lectures on the Automorphism Groups of Kobayashi-Hyperbolic Manifolds, Lecture Notes in Mathematics, 1902, Springer, Berlin, 2007. MR2352328 (2008j:32029)

[I2] Isaev, A. V., Hyperbolic 2-dimensional manifolds with 3-dimensional automorphism group, Geom. Topol. 12 (2008), 643-711. MR2403798(2009c:32048)

[K] Kobayashi, S., Hyperbolic Manifolds and Holomorphic Mappings. An Introduction, Second Edition, World Scientific Publishing Co. Pte. Ltd., Hackensack, NJ, 2005. MR2194466 (2006m:32008)

[L] Loeb, J.-J., Applications harmoniques et hyperbolicité de domaines tubes, Enseign. Math. (2) 53 (2007), 347-367. MR2455949 (2009g:32055)

[V] Vladimirov, V. S., Methods of the Theory of Functions of Many Complex Variables, The M.I.T. Press, Cambridge, Mass.-London, 1966. MR0201669 (34:1551)

Fakultät für Mathematik, Ruhr-Universität Bochum, Universitatstrasse 150, 44801 Bochum, Germany - and - School of Engineering and Science, Jacobs University Bremen, Campus Ring 1, 28759 Bremen, Germany

E-mail address: ahuck@gmx.de

Department of Mathematics, The Australian National University, Canberra, ACt 0200, Australia

E-mail address: alexander.isaev@anu.edu.au 